\title{
Study on the application effect of bisoprolol combined with sacubitril valsartan sodium tablets in the cardiac rehabilitation of patients with acute myocardial infarction combined with left heart failure after percutaneous coronary intervention (PCl)
}

\author{
Chi Chen ${ }^{1,2 \#}$, Xin $\mathrm{Wu}^{2,3 \#}$, Yufeng $\mathrm{Li}^{2,4}$, Yinian Peng ${ }^{2,4}$ \\ ${ }^{1}$ Department of Cardiology, Sichuan Provincial People's Hospital, University of Electronic Science and Technology of China, Chengdu, China; \\ ${ }^{2}$ Chinese Academy of Sciences Sichuan Translational Medicine Research Hospital, Chengdu, China; ${ }^{3}$ Heart Failure Center, Sichuan Provincial \\ People's Hospital, University of Electronic Science and Technology of China, Chengdu, China; ${ }^{4}$ Department of Rehabilitation Medicine, Sichuan \\ Provincial People's Hospital, University of Electronic Science and Technology of China, Chengdu, China \\ Contributions: (I) Conception and design: C Chen, X Wu; (II) Administrative support: Y Peng; (III) Provision of study materials or patients: All \\ authors; (IV) Collection and assembly of data: All authors; (V) Data analysis and interpretation: All authors; (VI) Manuscript writing: All authors; (VII) \\ Final approval of manuscript: All authors. \\ \#These authors contributed equally to this work. \\ Correspondence to: Yufeng Li; Yinian Peng. Department of Rehabilitation Medicine, No.32 West Section 2, First Ring Road, Chengdu 610072, China. \\ Email: nrgxskhkyt5@sina.com; peyinian@163.com.
}

\begin{abstract}
Background: To analyze the application effect of bisoprolol combined with sacubitril valsartan sodium tablets in the cardiac rehabilitation of patients with acute myocardial infarction combined with left heart failure after percutaneous coronary intervention (PCI).

Methods: Patients were divided into a control group (bisoprolol), with 39 cases, and a combination group (bisoprolol combined with sacubitril valsartan sodium tablets), with 42 cases, according to their different medications after surgery. The 6-min walking test distance, heart rate, oxygen saturation, serological indicators, cardiac function, incidence of cardiac adverse events, and adverse reactions of patients were compared between the 2 groups after treatment.
\end{abstract}

Results: The 6-min walking distance of the combination group was significantly higher than that of the control group, the heart rate was lower than that of the control group $(\mathrm{P}<0.05)$, and there was no difference in oxygen saturation $(\mathrm{P}>0.05)$. The levels of $\mathrm{N}$-terminal pro-B-type natriuretic peptide (NT-proBNP), intercellular adhesion molecule-1 (ICAM-1), and aldosterone (ALD) after treatment in the combination group were significantly lower than those in the control group $(\mathrm{P}<0.05)$. After treatment, the left ventricular end diastolic diameter (LVEDD) and left ventricular end systolic diameter (LVESD) of the combination group were significantly lower than those of the control group, and the left ventricular ejection fraction (LVEF) was significantly higher $(\mathrm{P}<0.05)$. There was no difference in left ventricular posterior wall thickness (LVPWT) level $(\mathrm{P}>0.05)$. The proportion of normal diastolic function in the combination group was $78.57 \%$, which was significantly higher than $43.59 \%$ in the control group, and the proportion of grade II was significantly lower than that in the control group $(4.76 \%$ vs. 25.64\%) $(\mathrm{P}<0.05)$. The total incidence of adverse cardiac events 6 months after treatment in the combination group was $9.52 \%$, which was significantly lower than $25.364 \%$ in the control group $(\mathrm{P}<0.05)$. There were no deaths in the 2 groups, and there was no difference in adverse reactions $(\mathrm{P}>0.05)$.

Conclusions: Bisoprolol combined with sacubitril valsartan sodium tablets has a good application effect in the cardiac rehabilitation of patients with acute myocardial infarction combined with left heart failure after PCI. It can promote cardiac rehabilitation and improve cardiac function, and reduce the incidence of cardiac adverse events. 


\begin{abstract}
Keywords: Bisoprolol; sacubitril and valsartan sodium; acute myocardial infarction with left heart failure; percutaneous coronary intervention (PCI); cardiac rehabilitation
\end{abstract}

Submitted Mar 18, 2021. Accepted for publication Apr 28, 2021.

doi: $10.21037 / \mathrm{apm}-21-877$

View this article at: http://dx.doi.org/10.21037/apm-21-877

\section{Introduction}

Acute myocardial infarction is caused by severe a reduction or interruption of coronary blood supply and severe myocardial ischemia causing myocardial necrosis. In clinical practice, patients often present with severe chest pain. Laboratory examinations show a significant increase in serum myocardial enzymes and abnormal electrocardiograms (1). Acute myocardial infarction is often accompanied by arrhythmia, heart failure, and other conditions. In severe cases, sudden death will occur, threatening the lives of high-risk patients (2).

Relevant studies have found that ventricular remodeling is involved in the occurrence and development of heart failure and is an important process of its pathological changes. Excessive excitation of the renin, angiotensin, and aldosterone systems is an important physiological mechanism that causes ventricular structural changes and disease progression (3). At present, enkephalinase inhibitors are often used as the first choice drug in the clinical treatment of heart failure, and its representative drug is sacubitril valsartan sodium tablets. Beta-blockers have pharmacological effects such as lowering blood pressure, controlling heart rate, and relieving angina pectoris. The combined use of the 2 has achieved good clinical effects in the treatment of heart failure, however, there are few studies on the cardiac rehabilitation of patients with acute myocardial infarction and left heart failure after percutaneous coronary intervention (PCI) $(4,5)$. Therefore, this article aims to analyze the effect of bisoprolol combined with sacubitril valsartan sodium tablets in the cardiac rehabilitation of patients with acute myocardial infarction and left heart failure after PCI. We present the following article in accordance with the STROBE reporting checklist (available at http://dx.doi.org/10.21037/apm-21-877).

\section{Methods}

\section{General information}

The clinical data of 81 patients with acute myocardial infarction and left heart failure admitted to our hospital from July 2018 to June 2020 were collected. All patients agreed to participate in this study and signed an informed consent form. The study was conducted in accordance with the Declaration of Helsinki (as revised in 2013). This study was approved by the Sichuan Provincial People's Hospital, University of Electronic Science and Technology of China (No. 20180615). The inclusion criteria were as follows: (I) All patients were treated with PCI; (II) all patients were treated within 6 hours after the onset of disease; (III) clinical data and imaging data during the treatment period were complete and there was no missing data; (IV) followup for 6 months; (V) did not receive $\beta$-blocker treatment 1 week before surgery. The exclusion criteria were as follows: (I) atrial fibrillation; (II) patients unable to perform the 6-minute walk test; (III) patients who were allergic to related treatment drugs; (IV) patients who had prior myocardial infarction; (V) patients who had severe valvular heart disease; (VI) patients who could not tolerate surgery. According to the different medications after surgery, patients were divided into the control group $(n=39)$ and the combination group $(n=42)$. In the control group, there were 24 males and 15 females, aged 39-70 years old, with an average age of $51.30 \pm 6.21$ years. Among these patients, there were 8 cases of diabetes, 13 cases of hypertension, and 18 cases of dyslipidemia. In the combination group, there were 27 males and 15 females, aged 39-70 years old, with an average age of $51.28 \pm 6.27$ years. Among these patients, there were 10 cases of diabetes, 14 cases of hypertension, and 18 cases of dyslipidemia. There were no statistically significant differences between the 2 groups of patients in regard to general data such as age, gender, and comorbidities $(\mathrm{P}>0.05)$, and they were comparable.

\section{PCI treatment}

After PCI treatment, all patients were treated with antiplatelet therapy and plaque stabilization according to the treatment guidelines and the patients' own conditions. On the basis of this treatment, patients in the control group 
Table 1 Comparison of 6-min walking distance, heart rate, and oxygen saturation before and after treatment between the 2 groups

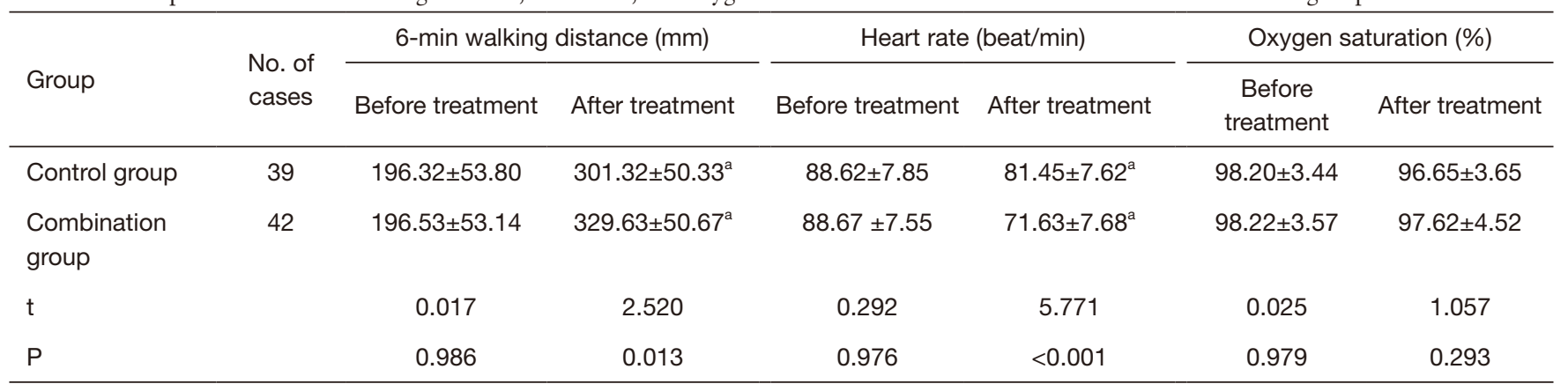

Compared with before and after treatment between the 2 groups, ${ }^{\mathrm{a}} \mathrm{P}<0.05$.

were treated with bisoprolol (National Medicine Standard H20173037, specification: $5 \mathrm{mg}$, Yueyang Xinhuada Pharmaceutical Co., Ltd.) alone, $1.25 \mathrm{mg} /$ time, Once daily. The combination group was treated with sacubitril valsartan sodium tablets (National Medicine Standard J20190001, specification: 50mg, Beijing Novartis Pharmaceutical Co., Ltd.) along with the treatment of the control group. The initial dose was $50 \mathrm{mg} /$ time, 2 times/d. After that, the dose was increased by $50 \mathrm{mg}$ every 2 weeks until $200 \mathrm{mg}$ was reached each time. Both groups of patients were treated for 4 consecutive weeks.

\section{Observation indicators}

(I) 6-min walking distance test (6): the patient's 6-min walking distance before and after treatment was measured. The longer the walk, the better the patient's recovery.

(II) Serum indicators: the indicators measured were $\mathrm{N}$-terminal pro-B-type natriuretic peptide (NTproBNP), intercellular adhesion molecule-1 (ICAM-1), and aldosterone (ALD). Before and after treatment, all patients were subjected to venous blood sampling, and the supernatant was obtained after centrifugation. Enzyme-linked immunosorbent assay was used for detection, and the test steps were carried out in accordance with the instructions provided with the reagents.

(III) Heart function was checked by echocardiography before and after treatment. The inspection instrument was the CXD-1 echocardiograph provided by Shenzhen Jiahaoke Electronic Technology Co., Ltd. The cardiac function indicators included left ventricular end diastolic diameter (LVEDD), left ventricular end systolic diameter (LVESD), left ventricular posterior wall thickness (LVPWT), left ventricular ejection fraction (LVEF), and patient stretch function. Diastolic work classifications were as follows: $\mathrm{EF}>40 \%$ was normal, left ventricular filling was falsely normalized to I degree, reversible filling limitation was II degree, and late irreversible filling limitation was III degree.

(IV) Adverse cardiac events: patients were followed up for 6 months to record the occurrence of non-fatal myocardial infarction, readmission due to heart failure, and death.

(V) Adverse reactions: hypotension, dizziness, angioedema, etc.

\section{Statistical methods}

The data in this study were statistically analyzed using SPSS22.0 software. The measurement data were described by the mean \pm standard deviation. The $t$-test was used for comparisons between pairs, and the variance test was used for comparisons between multiple groups. The count data were expressed by the pass rate or composition ratio, and the $\chi^{2}$ test was used. The differences were statistically significant with $\mathrm{P}<0.05$.

\section{Results}

Comparison of 6-min walking distance, heart rate, and oxygen saturation before and after treatment between the 2 groups

As shown in Table 1, before treatment, there was no difference in 6-min walking distance, heart rate, and oxygen saturation between the 2 groups of patients $(\mathrm{P}>0.05)$. However, the 6-min walking distance and heart rate 
Table 2 Comparison of serum indexes before and after treatment in the 2 groups

\begin{tabular}{|c|c|c|c|c|c|c|c|}
\hline Group & No. of cases & \multicolumn{2}{|c|}{ NT-proBNP $\left(\mathrm{pg} \cdot \mathrm{mL}^{-1}\right)$} & \multicolumn{2}{|c|}{ ICAM-1 (ng $\left.\mathrm{mL}^{-1}\right)$} & \multicolumn{2}{|c|}{ ALD $\left(p g \cdot \mathrm{mL}^{-1}\right)$} \\
\hline Control group & 39 & $692.62 \pm 48.62$ & $510.52 \pm 38.62^{\mathrm{a}}$ & $64.30 \pm 5.22$ & $43.65 \pm 3.82^{\mathrm{a}}$ & $233.62 \pm 35.10$ & $191.36 \pm 29.63^{a}$ \\
\hline $\begin{array}{l}\text { Combination } \\
\text { group }\end{array}$ & 42 & $693.11 \pm 48.24$ & $410.30 \pm 38.88^{\mathrm{a}}$ & $64.27 \pm 5.37$ & $31.95 \pm 3.18^{\mathrm{a}}$ & $230.62 \pm 35.67$ & $166.30 \pm 29.33^{a}$ \\
\hline
\end{tabular}

Compared with before and after treatment between the 2 groups, ${ }^{a} \mathrm{P}<0.05$. NT-proBNP, N-terminal pro-B-type natriuretic peptide; ICAM-1, intercellular adhesion molecule-1; ALD, aldosterone.

Table 3 Comparison of LVEDD, LVESD, LVPWT, and LVEF after treatment between the 2 groups

\begin{tabular}{|c|c|c|c|c|c|}
\hline Group & No. of cases & LVEDD (mm) & LVESD (mm) & LVPWT (mm) & LVEF (\%) \\
\hline Before treatment & & $50.72 \pm 5.43$ & $35.61 \pm 6.70$ & $9.63 \pm 1.62$ & $45.30 \pm 3.24$ \\
\hline After treatment & & $45.61 \pm 3.65^{a}$ & $32.32 \pm 4.61^{a}$ & $10.45 \pm 1.38$ & $51.30 \pm 3.68^{a}$ \\
\hline Combination group & 42 & & & & \\
\hline After treatment & & $40.30 \pm 3.11^{\mathrm{ab}}$ & $30.07 \pm 4.05^{\mathrm{ab}}$ & $10.52 \pm 1.51$ & $56.10 \pm 3.47^{\mathrm{ab}}$ \\
\hline
\end{tabular}

Compared with after treatment between the 2 groups, ${ }^{a} \mathrm{P}<0.05$; compared with before treatment within the group, ${ }^{\mathrm{b}} \mathrm{P}<0.05$. LVEDD, left ventricular end diastolic diameter; LVESD, left ventricular end systolic diameter; LVPWT, left ventricular posterior wall thickness; LVEF, left ventricular ejection fraction.

after treatment were improved $(\mathrm{P}<0.05)$, but there was no difference in oxygen saturation compared with before treatment $(\mathrm{P}>0.05)$. The 6-min walking distance after treatment in the combination group was significantly higher than that of the control group, the heart rate was lower than that of the control group $(\mathrm{P}<0.05)$, and there was no difference in oxygen saturation $(\mathrm{P}>0.05)$.

\section{Comparison of serum indexes before and after treatment in the 2 groups}

As shown in Table 2, there were no differences in the levels of serum NT-proBNP, ICAM-1, and ALD between the 2 groups of patients before treatment $(\mathrm{P}>0.05)$. After treatment, the levels of NT-proBNP, ICAM-1, and ALD were down-regulated compared with before treatment. In addition, the levels of NT-proBNP, ICAM-1, and ALD in the combination group were significantly lower than those in the control group after treatment. The difference between the 2 groups was statistically significant $(\mathrm{P}<0.05)$.

\section{Comparison of cardiac function between the 2 groups before and after treatment}

\section{Comparison of LVEDD, LVESD, LVPWT, and LVEF} between the 2 groups before and after treatment

As shown in Table 3, before treatment, there were no differences in LVEDD, LVESD, LVPWT, and LVEF between the 2 groups of patients $(\mathrm{P}>0.05)$. After treatment, the levels of LVEDD and LVESD were down-regulated, the levels of LVEF were up-regulated, and the levels of LVPWT remained unchanged. After treatment in the combination group, the levels of LVEDD and LVESD were significantly lower compared with the control group, the levels of LVEF were significantly higher $(\mathrm{P}<0.05)$, and there was no difference in the levels of LVPWT $(\mathrm{P}>0.05)$.

\section{Comparison of the diastolic function of the 2 groups of patients after treatment}

As shown in Table 4, the III degree did not occur in the 2 groups after treatment. The proportion of normal diastolic function in the combination group was $78.57 \%$, which was 
Table 4 Comparison of the diastolic function of the 2 groups of patients after treatment

\begin{tabular}{|c|c|c|c|c|c|}
\hline Group & No.of cases & Normal, n (\%) & I degree, n (\%) & II degree, n (\%) & III degree, n (\%) \\
\hline Combination group & 42 & $33(78.57)$ & $7(16.67)$ & $2(4.76)$ & $0(0.00)$ \\
\hline$\chi^{2}$ & & 10.475 & 2.240 & 6.985 & \\
\hline$P$ & & 0.001 & 0.134 & 0.008 & \\
\hline
\end{tabular}

Table 5 Comparison of adverse cardiac events and adverse reactions between the 2 groups of patients 6 months after treatment

\begin{tabular}{|c|c|c|c|c|c|c|c|c|c|}
\hline Group & $\begin{array}{l}\text { No. of } \\
\text { cases }\end{array}$ & \multicolumn{4}{|c|}{ Adverse cardiac events, n (\%) } & \multicolumn{4}{|c|}{ Adverse reactions, $\mathrm{n}(\%)$} \\
\hline Control group & 39 & $4(10.25)$ & $6(15.38)$ & 0 & $10(25.64)$ & $1(2.56)$ & $1(2.56)$ & 0 & $2(5.12)$ \\
\hline $\begin{array}{l}\text { Combination } \\
\text { group }\end{array}$ & 42 & $1(2.38)$ & $3(7.14)$ & 0 & $4(9.52)$ & $1(2.38)$ & $1(2.38)$ & $1(2.38)$ & $3(7.14)$ \\
\hline$\chi^{2}$ & & & & & 4.677 & & & & 0.142 \\
\hline$P$ & & & & & 0.031 & & & & 0.707 \\
\hline
\end{tabular}

significantly higher than that of the control group (43.59\%). The proportion of degree II was significantly lower than that of the control group ( $4.76 \%$ vs. $25.64 \%)$. The difference between the 2 groups was statistically significant $(\mathrm{P}<0.05)$.

\section{Comparison of adverse cardiac events and adverse reactions between the 2 groups of patients 6 months after treatment}

As shown in Table 5, 6 months after treatment, the total incidence of adverse cardiac events in the combination group was $9.52 \%$, which was significantly lower than that in the control group $(25.364 \%)(\mathrm{P}<0.05)$, and there were no deaths. However, there was no difference in adverse reactions between the 2 groups $(\mathrm{P}>0.05)$.

\section{Discussion}

In previous clinical treatments, it was found that revascularization of the patient's myocardium can induce local inflammation, and can cause cell apoptosis and myocardial calcium imbalance, which can lead to myocardial ischemia and reperfusion, resulting in myocardial stunning (7). Surgical trauma, inflammation, and cardiac arrest will adversely affect the recovery of patients after PCI $(8,9)$. Therefore, how to perform cardiac rehabilitation after PCI is one of the current clinical research hotspots.

Relevant studies have shown that the use of $\beta$-blockers in patients after PCI can reduce the mortality rate and the incidence of adverse cardiac events, and help patients recover (10). However, $\beta$-blockers can cause certain adverse effects on the cardiopulmonary function of patients. Patients are prone to fatigue and dizziness. In severe cases, they may experience difficulty breathing or even bradycardia. Based on this influence, the use of the drug in clinical practice is greatly restricted (11). As a $\beta$-blocker with high selectivity and affinity, bisoprolol can selectively $\beta$-blockers without affecting $\beta 2$ receptors. Its selectivity is significantly better than metoprolol, atenolol, and other drugs $(12,13)$. However, some studies have found that bisoprolol alone cannot achieve the expected results in the treatment of patients during rehabilitation after PCI (14). Angiotensin-converting enzyme inhibitor drugs can significantly improve heart function in patients with chronic congestive heart failure. Since most of the AT1 in the heart tissue is produced by the ACE pathway, angiotensinconverting enzyme inhibitor drugs cannot block this part of the effect of angiotensin II (15). As an angiotensin II receptor antagonist, sacubitril valsartan sodium tablets can selectively act on AT1, thereby blocking angiotensin II to achieve the 
purpose of treatment $(16,17)$. According to studies by related researchers, this drug can inhibit myocardial fibrosis and myocardial hypertrophy, thereby improving hemodynamics and cardiac function in patients with cardiomyopathy. It has a short-term effect on blood circulation and a long-term effect on local tissues. It has an inhibitory effect on vascular factors and norepinephrine, increases renal blood flow, and reduces aldosterone secretion, thereby reducing blood pressure and reducing the burden on the patient's heart. It can have long-term stable therapeutic effects, and is more suitable for long-term application (18).

In this study, the combination of bisoprolol and sacubitril valsartan sodium tablets was used in the cardiac rehabilitation of patients with acute myocardial infarction and left heart failure after PCI. It was found that this combination is effective and can better control the heart rate of patients, compared to those treated with bisoprolol alone. The 6-min walking distance and heart rate of patients in the combination group were significantly improved. At the same time, the improvement of LVEDD, LVESD, and LVEF indexes of the combination group was significantly better than that of the control group, and the normal rate of diastolic function was higher than that of the control group. NT-proBNP is an objective biological indicator of heart function, and has high value in the diagnosis and treatment of heart failure and prognostic evaluation (19). As a pro-inflammatory factor, ICAM-1 can cause thrombosis, leading to myocardial ischemia and heart failure (20). ALD is an effective factor in the differential diagnosis of heart failure, and it also has a certain value in judging the prognosis of patients (21). The levels of the 3 heart failure markers, NT-proBNP, ICAM1 , and ALD, were significantly reduced after the combined treatment, suggesting that the combination of the 2 drugs has an ideal clinical effect and can improve the heart failure of patients at the molecular level. Moreover, the incidence of adverse cardiac events at 6 months after the combined treatment was significantly lower than that of the single treatment, and it did not increase the adverse reactions.

In summary, bisoprolol combined with sacubitril valsartan sodium tablets has a good effect in the cardiac rehabilitation of patients with acute myocardial infarction and left heart failure after PCI, and can promote the patient's cardiac rehabilitation, improve cardiac function, and reduce cardiac deficiencies.

\section{Acknowledgments}

Funding: None.

\section{Footnote}

Reporting Checklist: The authors have completed the STROBE reporting checklist. Available at http://dx.doi. org/10.21037/apm-21-877

Data Sharing Statement: Available at http://dx.doi. org/10.21037/apm-21-877

Conflicts of Interest: All authors have completed the ICMJE uniform disclosure form (available at http://dx.doi. org/10.21037/apm-21-877). The authors have no conflicts of interest to declare.

Ethical Statement: The authors are accountable for all aspects of the work in ensuring that questions related to the accuracy or integrity of any part of the work are appropriately investigated and resolved. This study was approved by the Sichuan Provincial People's Hospital, University of Electronic Science and Technology of China (No. 20180615). All patients agreed to participate in this study and signed an informed consent form. The study was conducted in accordance with the Declaration of Helsinki (as revised in 2013).

Open Access Statement: This is an Open Access article distributed in accordance with the Creative Commons Attribution-NonCommercial-NoDerivs 4.0 International License (CC BY-NC-ND 4.0), which permits the noncommercial replication and distribution of the article with the strict proviso that no changes or edits are made and the original work is properly cited (including links to both the formal publication through the relevant DOI and the license). See: https://creativecommons.org/licenses/by-nc-nd/4.0/.

\section{References}

1. Tomoaia R, Beyer RS, Simu G, et al. Understanding the role of echocardiography in remodeling after acute myocardial infarction and development of heart failure with preserved ejection fraction. Med Ultrason 2019;21:69-76.

2. Kim SH, Cho JH, Lee YH, et al. Improvement in Left Ventricular Function with Intracoronary Mesenchymal Stem Cell Therapy in a Patient with Anterior Wall STSegment Elevation Myocardial Infarction. Cardiovasc Drugs Ther 2018;32:329-38.

3. Xu M, Yan L, Xu J, et al. Predictors and prognosis for incident in-hospital heart failure in patients with preserved 
ejection fraction after first acute myocardial infarction: An observational study. Medicine (Baltimore) 2018;97:e11093.

4. Sygitowicz G, Maciejak-Jastrz bska A, Sitkiewicz D. MicroRNAs in the development of left ventricular remodeling and postmyocardial infarction heart failure. Pol Arch Intern Med 2020;130:59-65.

5. Li J, Cai SX, He Q, et al. Intravenous miR-144 reduces left ventricular remodeling after myocardial infarction. Basic Res Cardiol 2018;113:36.

6. Kotecha D, Bunting KV, Gill SK, et al. Effect of Digoxin vs Bisoprolol for Heart Rate Control in Atrial Fibrillation on Patient-Reported Quality of Life: The RATE-AF Randomized Clinical Trial. JAMA 2020;324:2497-508.

7. Tsutsui H, Momomura SI, Masuyama T, et al. Tolerability, Efficacy, and Safety of Bisoprolol vs. Carvedilol in Japanese Patients With Heart Failure and Reduced Ejection Fraction- The CIBIS-J Trial. Circ J 2019;83:1269-77.

8. Kishi T, Fujii E. Carvedilol and bisoprolol as initial therapy for adult hypertension without compelling indications. Hypertens Res 2019;42:496-503.

9. Imori Y, Takano H, Mase H, et al. Bisoprolol transdermal patch for perioperative care of non-cardiac surgery in patients with hypertrophic obstructive cardiomyopathy. BMC Cardiovasc Disord 2019;19:316.

10. Fernandez-Lopez L, Pellegrini M, Rotolo MC, et al. Development and Validation of a Method for the Analysis of Bisoprolol and Atenolol in Human Bone. Molecules 2019;24:2400.

11. Solomon SD, Jhund PS, Claggett BL, et al. Effect of Dapagliflozin in Patients With HFrEF Treated With Sacubitril/Valsartan: The DAPA-HF Trial. JACC Heart Fail 2020;8:811-8.

12. Uijl E, MirabitoColafella KM, Sun Y, et al. Strong and Sustained Antihypertensive Effect of Small Interfering RNA Targeting Liver Angiotensinogen. Hypertension 2019;73:1249-57.

13. Liu Z, Wang J, Li Y. Efficacy of sacubitril valsartan sodium tablet for the treatment of chronic heart failure: A systematic review protocol of randomized controlled trials.

Cite this article as: Chen $\mathrm{C}, \mathrm{Wu} \mathrm{X}, \mathrm{Li}$ Y, Peng Y. Study on the application effect of bisoprolol combined with sacubitril valsartan sodium tablets in the cardiac rehabilitation of patients with acute myocardial infarction combined with left heart failure after percutaneous coronary intervention (PCI). Ann Palliat Med 2021;10(5):5455-5461. doi: 10.21037/apm-21-877
Medicine (Baltimore) 2019;98:e18050.

14. Hamano G, Yamamoto K, Takami Y, et al. Effects of Low-Dose Sacubitril/Valsartan on Different Stages of Cardiac Hypertrophy in Salt-Loaded Hypertensive Rats. J Cardiovasc Pharmacol 2019;73:282-9.

15. Yang K, Wang Y, Ding Y, et al. Valsartan chronotherapy reverts the non-dipper pattern and improves blood pressure control through mediation of circadian rhythms of the renin-angiotensin system in spontaneous hypertension rats. Chronobiol Int 2019;36:1058-71.

16. Wadell D, Jensen J, Englund E, et al. Triple therapy after PCI - Warfarin treatment quality and bleeding risk. PLoS One 2018;13:e0209187.

17. Kalinauskiene E, Gerviene D, Bacharova L, et al. Differences in the Selvester QRS score after primary PCI strategy and conservative treatment for STEMI patients with negative T waves. Ann Noninvasive Electrocardiol 2019;24:e12684.

18. Zheng $\mathrm{X}, \mathrm{Wu} \mathrm{L}$. The value of cytochrome P4502C19 gene assay for anti-platelet therapy after PCI in stable angina patients with left main coronary artery lesions. Nan Fang Yi Ke Da Xue Xue Bao 2020;40:274-8.

19. Chen GX, Wang HN, Zou JL, et al. Effects of intracoronary injection of nicorandil and tirofiban on myocardial perfusion and short-term prognosis in elderly patients with acute ST-segment elevation myocardial infarction after emergency PCI. World J Emerg Med 2020;11:157-63.

20. Guo W, Su Y, Chen L, et al. Effects of nursing methods for emergency PCI and non-emergency PCI on the treatment of patients with acute myocardial infarction. J Pak Med Assoc 2020;70:31-7.

21. Roule V, Schwob L, Briet C, et al. Residual platelet reactivity, thrombus burden and myocardial reperfusion in patients treated by PCI after successful pre-hospital fibrinolysis compared to primary PCI. J Thromb Thrombolysis 2020;50:858-66.

(English Language Editor: C. Betlazar-Maseh) 